\title{
Kolona metalik stent yerleştirilmesi; 7 yıllık deneyim
}

\section{Colonic self-expandable metal stent placement: Seven-year experience}

Erkin ÖZTAŞ ${ }^{1}$, Muhammet Yener AKPINAR ${ }^{2}$, Selçuk DIŞIBEYAZZ1 ${ }^{1}$ Bülent ÖDEMIŞ33 Erkan PARLAK $^{4}$

Eskişehir Osmangazi Universitesi Tip Fakültesi, ${ }^{1}$ Iç Hastalkklan Anabilim Dalı, Gastroenteroloji Bilim Dalı, Eskişehir

Keçiören Eğitim ve Araştırma Hastanesi, ${ }^{2}$ Gastroenteroloji Kliniği, Ankara

Türkiye Yüksek Ihtisas Eğitim ve Araștırma Hastanesi, ${ }^{3}$ Gastroenteroloji Kliniği, Ankara

Hacettepe Üniversitesi Tıp Fakültesi, ${ }^{4} I_{c ̧}$ Hastalıklan Anabilim Dall, Gastroenteroloji Bilim Dalı, Ankara

Giris ve Amaç: Kendiliğinden genişleyebilen metalik stentler kolonun benign ve malign bir çok hastalığında giderek artan sıklıkta kullanılmaktadır. Malign ve benign darlıklar, kaçaklar ve perforasyonlar en sık görülen kullanım endikasyonlarıdır. Bu çalışmada amacımız kolona kendiliğinden genişleyebilen metalik stent yerleştirmiş olduğumuz hastaların verilerini retrospektif olarak değerlendirmektir. Gereç ve Yöntem: Türkiye Yüksek Ihtisas Eğitim ve Araștırma Hastanesinde 2010-2017 yılları arasında kolona kendiliğinden genişleyebilen metalik stent yerleştirilen hastaların verileri retrospektif olarak incelendi. Hastalara kolon stentleri 2 farklı teknik kullanılarak yerleştirildi. Distal lezyonlarda kılavuz tel üzerinden yalnızca floroskopi yardımıyla, proksimal lezyonlarda ise kolonoskopun terapötik kanalından kendiliğinden genişleyebilen metalik stent geçirilerek endoskop ve floroskop birlikte kullanılarak yerleștirildi. Bulgular: Çalışmaya toplam 41 hasta alınd (yaş ortancası: 63,6, erkek/kadın: 28/13). 40 hastaya kolonik obstrüksiyon, 1 hastaya ise anastomoz kaçağı nedeniyle kendiliğinden genişleyebilen metalik stent yerleştirildi. Kolonik obstrüksiyonu olan hastaların 36 tanesinde neden kolorektal kanserken diğer 4 hasta benign nedenlere bağlı darlık gelişmiști. Genel olarak bakıldığında teknik bașarı oranı \%82, klinik bașanı oranı ise \%96 olarak izlendi. Toplam 4 hastada komplikasyon geliştiği görüldü iki hastada erken dönemde perforasyon ve migrasyon geliști. Diğer iki hastada geç dönemde reobstrüksiyon geliştiği görüldü. Tartışma: Kolonik metal stentlerin yüksek teknik ve klinik başarıları ve düsük komplikasyon oranları, bu stentleri uygun endikasyonu olan hastalarda önemli bir tedavi modalites haline getirmiştir. Bu nedenle sadece kolorektal kanserde değil, anastomoz darlıklarında ve kaçaklarında da kolonik metal stentlerin önemli bir tedavi metodu olduğu akılda tutulmalıdır.

Anahtar kelimeler: Kolon, SEMS

\section{GİRIS}

Enteral stentler; perforasyon, fistül ve kaçak gibi durumlarda lümenin bütünlügünün idamesi veya intralüminal darlık veya ekstralüminal bası gibi durumlarda yeniden sağlanması amaclyla kullanılırlar (1,2). Kolona stent denemeleri ilk kez 1986 yllında Lelcuk ve arkadaşlarının sol kolon malign obstrüksiyonunda nazogastrik tüp ile dekompresyonu denemeleri ile başlamıs ve 1992 yılında Spinelli ve arkadaşlarının rektal malign obstrüksiyonda "self expanding mesh stent" kullanımıyla yeni bir tedavi metodu olarak karşımıza çıkmışır (3).

Son 2-3 dekatta ise gastrointestinal sisteminde "self expandable metallic stent" (SEMS) kullanımı giderek artmış ve buna paralel olarak stent teknolojisinde kayda değer gelişmeler ol-
Background and Aims: Self-expandable metal stents are being used in both benign and malignant colonic disease with increased frequency. Benign and malignant obstructions, leakages, and/or perforations are the main clinical indications for colonic self-expandable metal stents. Here we evaluated our colonic self-expandable metal stents patients retrospectively. Materials and Methods: Patients in whom colonic self-expandable metal stents were inserted between 2010 and 2017 were examined retrospectively. Colonic self-expandable metal stents were inserted by two different techniques. For distal lesions, self-expandable metal stents were inserted over the guide-wire with assistance of fluoroscopy; in proximal lesions, self-expandable metal stents were inserted with combined assistance of both colonoscopy and fluoroscopy. Results: A total of 41 patients were enrolled in the study (median age: 63.6 years, males/females: 28/13). Self-expandable metal stents were inserted in 40 patients because of a colonic obstruction, and in one patient because of an anastomotic leakage. Thirty-six patients had colorectal cancer, and 4 patients had benign disease-related obstructions. Our technical success rate was $82 \%$, and our clinical success rate was 96\%. Complications were observed in 4 patients: one perforation and one migration occurred during the early period. In 2 patients re-obstruction occurred during the late period. Conclusion: With high technical and clinical success and low complication rates, self-expandable metal stents are a valuable treatment modality in appropriate patients. Therefore, it is important to remember that insertion of colonic self-expandable metal stent is an effective treatment method in not only colorectal malignancies but also anastomotic stenosis and leakage.

Key words: Colon, self-expandable metal stents

muştur. SEMS'ler; kapsız, tam kaplı ve parsiyel kaplı olmak üzere üç çeşittir. Tam kaplı olanların iç yüzeyi tamamen silikon veya plastik membranla kaplıdır. Parsiyel kaplı olanlarda bu durum yalnız gövdede söz konusudur. Paslanmaz çelikten yapıldıkları gibi -ki günümüzde nadiren kullanılmaktadırlar-, çeşitli metallerin karışımdan da yapılmaktadırlar. Elgiloy; kobalt, nikel ve krom alaşımından oluşurken, nitinol nikel ve titanyum alaşımından oluşur. Nitinol SEMS'ler, Elgiloy yapıdaki SEMS'lere göre daha esnektir, eşsiz şekil hafızası mevcuttur ve MR uyumludur. SEMS'ler yalnızca floroskopi yardımıyla yerleştirilebilmeleri mümkün olmakla birlikte bugün için endoskopi ve floroskopi kombinasyonu altında yerleştirilmeleri 
önerilmektedir. SEMS'ler endoskopun terapotik kanalından geçirilerek yerleştirilebildikleri gibi ["Through the Scope Technique", (TTST)], endoskop yardımı olmaksızın floroskopik olarak kontrol edilerek klavuz tel yardımıyla ["Over the Wire Technique" (OTWT)] da yerleştirilebilirler (2).

Kapsız SEMS'lerde migrasyon riski düşük olmakla birlikte yüksek doku-tümor ingrowth riski söz konusudur. Kaplı SEMS'lerde bunun tam tersi geçerlidir. Parsiyel kaplı SEMS'ler ise kaplı SEMS'lere göre daha az migrasyon riski taşırlar ancak özellikle yerleştirilmelerinin üzerinden uzun süre geçmesi halinde çıkarılmaları zordur (1).

SEMS'lerin kolonda günümüze kadar en sık malign darlık endikasyonuyla kullanıldığı görülmektedir. Malign darlıklarda da cerrahiye köprü ve palyasyon amaçlı kullanılırlar. Diğer endikasyonlar; benign darlıklar (divertikülit ilişkili darlık, anastamoz darlığı, Crohn hastalığına bağlı darlık, radyoterapiye bağlı darlık, iskemik darlık), eksternal basıya bağlı darlık ve fistül-kaçaklardır. Literatürde ancak birkaç olguda iatrojenik perforasyonda kullanıldıkları görülmektedir (4-14).

Bu çalışmada amacımız kliniğimizde geçen 7 ylllık süreçte kolona SEMS yerleştirmiş olduğumuz hastaların verilerini retrospektif olarak değerlendirmektir.

\section{GEREC ve YÖNTEM}

Türkiye Yüksek Ihtisas Eğitim ve Araştırma Hastanesinde 2010-2017 yılları arasında kolona SEMS yerleştirilen hastalarin verileri retrospektif olarak incelendi. Hasta bilgilerine hasta dosyalarından, hastane bilgisayar otomasyon sisteminden ve hastaların aileleri ile iletişime geçilerek ulaşıldı.

\section{Hazırlık}

SEMS yerleştirilmesi için refere edilen veya primer olarak hastanemizde takip ve tedavi edilen hastaların dosyaları, klinik ve laboratuvar bulguları değerlendirilerek SEMS endikasyonu konulan hastalar çalışmaya alındı. SEMS yerleştirilmesi planlanan hastaların tamamına bir ya da iki kez rektal lavman uygulandı. Eğer endikasyon fistül/kaçak ise ve mutlaka tam barsak hazırlığı gerekecek ise işlem öncesi sennozid A+B veya polietilen glikol oral yolla verilerek uygun barsak temizliği sağlanılmaya çalışıldı. İşlemler EVIS LUCERA ELITE CLV290-SL (Olympus Medical Systems, Tokyo, Japan) model kolonoskop ile yapıldı. Kolon stenti yerleştirilmesi işlemi girişimsel endoskopik işlemler konusunda deneyimli uzman gastroenterologlar tarafindan yapıldı.

\section{Teknik}

Hastalara kolon stentleri 2 farklı teknik kullanılarak yerleştirildi. Distal lezyonlarda OTWT (Over the wire technique), proksimal lezyonlarda Through Scope Technique (TTST) uygulandı. Kısaca her iki teknikte de kolonoskop ile giri- lerek lezyona ulaşıldı (Resim 1A). Kolonoskopun terapötik kanalından, endoskopik retrograd kolanjiyopankreatografi (ERCP) taş çıkarma balonu yardımıyla, standart ERCP kılavuz teli (450 mm) geçirildi (Resim 1B). Bu tel lezyonun proksimaline geçirilmeye çalışıldı. Proksimale geçirilen kılavuz tel üzerinden yine tercihen ERCP taş balonu da geçirilerek bu balonun kanalından suda erir opak verildi ve striktrogram alındı veya kaçak görüntülendi (Resim 1C). Daha sonra kılavuz tel lezyonun proksimaline tekrar gönderildi. Lezyon hizasında, hastanın cildine çeşitli metaller yapıştırıldı ve floroskopik olarak da lokalizasyon işaretlendi. Distal lezyonlarda kilavuz tel lezyonun proksimalinde kalacak şekilde kolonoskop çıkarıldı ve bu kılavuz tel üzerinden genellikle yalnızca floroskopi kontrolüyle nadiren de arkasından kolonoskopla girilmek suretiyle endoskopi kontrolü de sağlanarak OTWT ile lezyona SEMS yerleştirildi. Proksimal lezyonlarda ise stent kolonoskopun terapötik kanalından geçirilerek lezyon seviyesinde açlldı (TTST) (Resim 1D, 1E). Stentler lezyonun boyundan en az $4 \mathrm{~cm}$ daha uzun olacak şekilde seçildi. Malign darlıklarda kapsız veya parsiyel kaplı SEMS'ler kullanılırken, benign durumlarda kaplı SEMS'ler tercih edildi.

Rektum tümörü nedeniyle rektosigmoidektomi, koloanal anastomoz ve loop ileostomi yapılmış bir olguda anastomoz hattı tamamen dağılmıştı. Bu hastada distalden stent yerleştirilmesi mümkün olmadığı için loop ileostominin kolonik tarafından girilerek kolonun en distal noktasına ulaşıldı. Stenotik bir uçla karşılaşıldı. Buradan klavuz tel gönderildiğinde telin anal kanalda beklenmedik bir noktadan dışarı çıktığı görülerek bu tel üzerinden randevu yöntemiyle transanal yolla OTWT kullanılarak kaplı SEMS yerleştirildi. Bu hastada SEMS'in distal 2 cm'lik kısmı anal kanaldan dışarı sarktı.

Özellikle darlıklarda işlem esnasında SEMS yerleştirilme öncesi veya sonrası balon dilatasyonu yapılıp yapılmadığı da kaydedildi.

Kullanılan stent tipi, uzunluğu, gövde genişliği de kaydedildi.

\section{İşlem Başarısı}

Hastalarda işlem başarısı; teknik başarı ve klinik başarı olarak iki alt başlıkta incelendi. Teknik başarı darlık veya anastomoz hattını ortalayacak şekilde stent yerleştirilmesi ve stentin başarılı bir şekilde açılması olarak tanımlandı. Klinik başarı ise malign darlık nedeniyle stent konulan hastalarda 24 saat içinde gaz ve gayta deşarjı olması olarak tanımlandı. Benign darlık veya kaçak da ise eğer stent belirli bir süre için konulmuş ise stent çıkarıldıktan sonra semptomların düzelmesi olarak ifade edildi.

\section{Komplikasyonlar}

Perforasyon, primer stent başarısızlığı, migrasyon, reobstrüksiyon başta olmak üzere gözlenen komplikasyonlar da kaydedildi. 

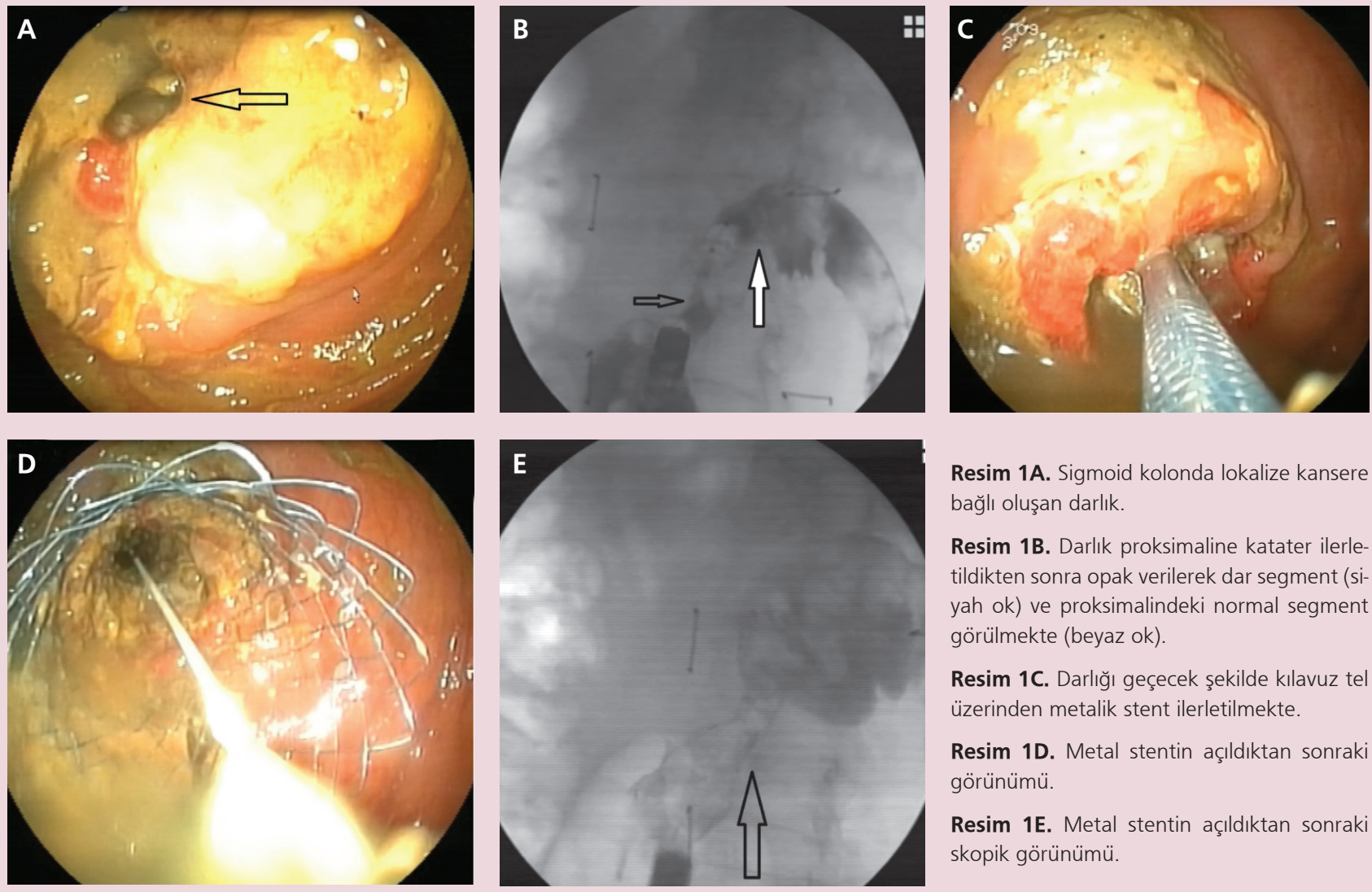

Resim 1A. Sigmoid kolonda lokalize kansere bağlı oluşan darlık.

Resim 1B. Darlık proksimaline katater ilerletildikten sonra opak verilerek dar segment (siyah ok) ve proksimalindeki normal segment görülmekte (beyaz ok).

Resim 1C. Darlığı geçecek şekilde kılavuz tel üzerinden metalik stent ilerletilmekte.

Resim 1D. Metal stentin açıldıktan sonraki görünümü.

Resim 1E. Metal stentin açıldıktan sonraki skopik görünümü.

\section{BULGULAR}

Çalışmaya 41 hasta alındı. Bu hastaların yaş ortancası 63,6 (4190) olup 13 hasta kadın, 28 hasta ise erkekti. 40 hastaya kolon obstrüksiyonu nedeniyle stent takılırken 1 hastada darlığa anastomozda geniş defekt oluşumu ve kaçak eşlik etmekteydi. Hastalarda kolon obstrüksiyonuna yol açan etiyolojik neden 36 hastada kolorektal kanserlerdi. 4 hastaya ise benign obstrüksiyon nedeniyle SEMS uygulanmıştı. Bu hastaların 3'ünde anastomoz darlığı (1 hasta opere rektum kanseri ve kaçak, 1 hasta opere volvulus, 1 hasta Crohn hastalığı'na bağlı ileotransversostomili), birinde ise mesane kanseri sonrası almış olduğu radyoterapiye bağlı oluşan darlık mevcuttu. Tüm hastalar bir arada değerlendirildiğinde stent uygulanan lokalizasyon en sık 20 hastayla sigmoid kolondaydı (Tablo 1). Anastomoz kaçağı ise rektumdaydı. Kolorektal kansere bağlı obstrüksiyon kliniği ile gelen hastalardan sadece 1 tanesine cerrahiye köprü amaçlı stent takılmıştı, diğer malign hastaların hepsine kolon stenti palyatif amaçla konuldu. 41 hastanın 33'ü takip süresince exitus oldu. Bu hastalarda stent takılması ile mortalite arasında geçen süre ortanca 192 (14-1449) gün olarak tespit edildi (Tablo 1).

Kırk bir hastaya toplam 45 adet metal stent takıldı. Crohn hastalığı nedeniyle ileotransversostomili olgumuza 6 ay arayla ardışık 2 adet metal stent takıldı. Kolorektal kanser tanılı 2 olguda darlık alanını tek stentin geçemeyeceği düşünül- dügünden iç içe 2 adet metal stent takılmıştı. Kolon kanseri tanılı 1 olgumuza ise ilk stentten 30 gün sonra obstrüksiyon kliniği geliştiği için 2. metal stent takılmıştı. Hastalarımıza takılan stentlerin özelliklerine bakıldığı zaman 42 tanesi kapsız, 1 tanesi yarı kaplı olup 2 tanesi ise tam kaplı metal stentlerdi. Metal stentlerin 44 tanesi standart teknikle takllırken 1 tanesi endoskopik randevu yöntemiyle takıldı. Balon dilatasyonu toplam 4 olguda uygulandi. Bunlardan 2 tanesinde balon dilatasyonu stent takılmadan önce yapılırken 2 tanesindeyse stent takıldıktan sonra balon dilatasyon uygulandı. 45 metal stentin 37 tanesi teknik olarak başarılı bir şekilde yerleştirildi (\%82). 8 işlem başarısızlıkla sonuçlandı. Teknik başarısızlık nedenlerine bakıldığı zaman 2 hastada yetersiz kolon temizliği nedeniyle alanın net vizualize edilememesi, 2 hastada stentin boyunu geçen uzun darlık ve 4 hastada ise denenmesine rağmen proksimale klavuz tel geçirilememesiydi. Hastaların 34'ünde izlem süresinde klinik başarı olup olmadığı değerlendirilebildi. Bu hastaların 33 tanesinde kolon stenti yerleştirildikten 24 saat sonra gaz ve gayta çıkışı olmuştu (\%96). Erken komplikasyon olarak 1 hastada (\%3) stent yerleştirilmesinden hemen sonra gelişen perforasyon, 1 hastada (\%3) ise stent migrasyonu saptandı. Ayrıca yine bahsedildiği üzere kolorektal kanser tanılı 1 ve Crohn hastalığı olan 1 hastada (toplam 2 hasta, \%6) sirasiyla 1. ve 6. ayda reobstrüksiyon geliştiği için tekrar stent konuldu (Tablo 2). 
Tablo 1. Hastaların epidemiyolojik özellikleri, kolon stenti endikasyonları ve mortalite oranları

\begin{tabular}{|c|c|}
\hline Özellikler & Sayı \\
\hline Hasta sayısı & 41 \\
\hline Yaş & $63,6(41-90)$ \\
\hline Cinsiyet (erkek/kadın) & $28 / 13$ \\
\hline \multicolumn{2}{|l|}{ Primer hastalık } \\
\hline 1. Kolorektal kanser & 36 \\
\hline \multicolumn{2}{|l|}{ 2. Anastomoz darlığı } \\
\hline a. Malignite operasyonu sonrası & 1 \\
\hline b. Crohn, ileotransversostomili & 1 \\
\hline c. Volvulus operasyonu sonrası & 1 \\
\hline 3. Radyoterapi darlığı & 1 \\
\hline 4. Anastomoz kaçağı & 1 \\
\hline \multicolumn{2}{|l|}{ Darlık veya kaçak lokalizasyonu } \\
\hline 1. Rektum & 15 \\
\hline 2. Sigmoid & 20 \\
\hline 3. Inen kolon & 2 \\
\hline 4. Splenik fleksura & 1 \\
\hline 5. Transvers & 3 \\
\hline \multicolumn{2}{|l|}{ Survey } \\
\hline 1. Mortalite var & 33 \\
\hline 2. Mortalite yok & 8 \\
\hline Stent takılma-ex zamanı arası süre (gün) & $192(4-1449)$ \\
\hline
\end{tabular}

\section{TARTIŞMA}

Kolorektal hastalıklarda metal stentlerin kullanım alanları ve hangi durumlarda metal stentlerden fayda görülebileceği son yıllarda yapılan çalışmalar ve yayınlanan kılavuzlarla daha iyi ortaya konulmuştur (1-4). Literatürde metal stentlerin kolorektal hastalıklarda en sık kullanıldığı alanın kolorektal kanserler olduğu görülmektedir (4). Bizim çalışmamızda da hastalarımızın çoğunu kolorektal malignitesi olan hastalar oluşturmaktaydı.

Kolonun malign darlıklarında SEMS yerleştirilmesinde teknik başarı \%46,7-100, klinik başarı ise \%92'dir (2,4). Bizim çalışmamızda ise tüm hastalar göz önüne alındı̆̆ında teknik başarımız \%82, klinik başarımız ise \%96 idi.

Literatür gözden geçirildiğinde stent yerleştirme esnasında mortalitenin \%0,6 olduğu görülmektedir. Erken komplikasyonlar <30 gün içinde olan, perforasyon \% 0-12,8 (Ortalama: $\% 7,4$ ), stent failure (açlamama) \%0-12.8, migrasyon \%0-4,9, reobstrüksiyon \%0-4,9, ağrı \%0-\%7,4, minör kanama \%03,7'dir. 30. gün mortalite $\% 9$ olup bunun yarısından stent ilişkili komplikasyonlar sorumludur. Geç komplikasyonlar (>30 gün); reobstrüksiyon (tümör ingrowth-overgrowth'u,
Tablo 2. Kolonik stent özellikleri, teknik ve klinik başarı oranları

\begin{tabular}{|c|c|}
\hline Özellikler & Sayı \\
\hline Stent sayısı & 45 \\
\hline $\begin{array}{l}\text { Metal stent özellikleri } \\
\text { 1. Kapsız } \\
\text { 2. Parsiyel kaplı } \\
\text { 3. Tam kaplı }\end{array}$ & $\begin{array}{c}42 \\
1 \\
2\end{array}$ \\
\hline $\begin{array}{l}\text { Stent takılma tekniği } \\
\text { 1. Standart (anal kanaldan tel üzerinden) } \\
\text { 2. Endoskopik randevu (proksimalden tel üzerinder }\end{array}$ & $\begin{array}{l}44 \\
\text { n) } 1\end{array}$ \\
\hline $\begin{array}{l}\text { Malignitesi olan hastalarda stent takılma endikasyonu } \\
\text { 1. Palyatif } \\
\text { 2. Cerrahiye köprü }\end{array}$ & $\begin{array}{c}35 \\
1\end{array}$ \\
\hline $\begin{array}{l}\text { Balon dilatasyon } \\
\text { 1. Stent öncesi } \\
\text { 2. Stent sonrası }\end{array}$ & $\begin{array}{l}2 \\
2\end{array}$ \\
\hline Teknik başarı & $37(\% 82)$ \\
\hline $\begin{array}{l}\text { Teknik başarısızlık nedenleri } \\
\text { 1. Kirli kolon } \\
\text { 2. Uzun darlık } \\
\text { 3. Sıkı darlık }\end{array}$ & $\begin{array}{l}2 \\
2 \\
4\end{array}$ \\
\hline $\begin{array}{l}\text { Klinik başarı } \\
\text { 1. Klinik başarı var } \\
\text { 2. Klinik başarı yok } \\
\text { 3. Değerlendirilemedi }\end{array}$ & $\begin{array}{c}33(\% 96)^{*} \\
1 \\
11\end{array}$ \\
\hline $\begin{array}{l}\text { Komplikasyon } \\
\text { 1. Perforasyon } \\
\text { 2. Migrasyon } \\
\text { 3. Reobstrüksiyon }\end{array}$ & $\begin{array}{l}1 \\
1 \\
2\end{array}$ \\
\hline
\end{tabular}

*; Klinik başarı ancak takip verilerine ulaşılabilen 34 hasta baz alınarak hesaplanabildi.

fekal impaksiyon) \%4-\%22.9, stent migrasyonu \%1-12.5, perforasyon \%0-4'dür. SEMS ilişkili nadir komplikasyonlar; tenesmus, inkontinans, fistül gelişimi olarak ifade edilmektedir. Gövde çapı $\geq 24$ mm kaydıyla; tam kaplı veya kapsız stentler birbirine eşit etkinlikte ve güvenilirliktedir. Her iki tür stentin avantajları ve dezavantajları birbirini dengelemektedir. Kapsız SEMS'de migrasyon \%5.5, tümör ingrowth'una bağlı obstrüksiyon \%11.4, kaplı SEMS'de migrasyon \%21.3, tümör ingrowth'una bağlı obstrüksiyon \%0.9'dur (4,5). Bizim olgularımızda komplikasyon olarak \%3 perforasyon, \%3 migrasyon ve \%6 reobstrüksiyon saptanmıştır. Takip verilerine ulaşabildiğimiz hastalara ait veriler göz önüne alındığında stent ilişkili mortalitenin hasta serimizde olmadığını söyleyebiliriz.

Anastomoz darlık ve kaçakları da kolonik metal stentlerin kullanım alanları arasındadır. Kolorektal cerrahi sonrası oluşan anastomoz kaçakları hastaların morbidite ve mortalitelerinde artışla beraberdir. Endoskopik olarak anastomoz 
kaçaklarının tedavisi son yıllarda daha sık başvurulan bir yöntem olmuştur (6-14). Bizim çalışmamızda bir hastaya anastomoz kaçağından dolayı metal stent konulmuştu. Bu hastamızın anastomozunun ileri derecede bozulmuş olması, anal yoldan metal stent konulmasını imkansız hale getirdiği için bu hastamıza literatürde çok az hasta için tarif edilen bir yöntem olan endoskopik randevu yöntemiyle stent konuldu. Burada hastanın loop ileostomisinde kolonoskop ile girilerek anastomoz hattının proksimaline ilerlendi ve kılavuz tel buradan geçip anal kanaldan çıkacak şekilde ilerletildi. Tel üzerinden de tam kaplı metal stent konularak anastomozdaki açıklık kapatıldı. 4 hafta sonra metal stent çıkarıldığında ise defektin tamamen kapandığı görüldü. Crohn hastalarında gelişen anastomoz darlıklarında da metalik stentleri kullanımı ile ilgili son dönemde yapılmış çalışmalar ve derlemeler mevcuttur. (10). Çalışmamızda kolonik metal stent konulan hastalardan biri Crohn hastasıydı ve ileotransversostomi sonrası anastomoz darlığı gelişmişti. Ileus kliniği ile gelen bu hastamızın aynı zamanda Child-B kriptojenik sirozu olduğu için cerrahi riski yüksek kabul edilerek endoskopik tedavi planlanmıştı. Anastomoz darlığına ilk seansta yarı kaplı metalik stent konulmuştu ve bu stentle hasta 6 ay obstrüktif bir şikayeti olmadan yaşamıştı. Altıncı ayda darlığı nüks eden hastaya 2. seansta da metalik stent konuldu.

Çalışmamızın en önemli eksikliği çalışmanın retrospektif yapılmış olmasıdır. Özellikle 11 hastanın takip ve klinik yanıt verilerine ulaşılamamıştır. Bunun yanında kullanılan stentlerin markaları, çaplanı ve uzunlukları ile ilgili de veriler eksiktir. Diğer taraftan çalışmamızda bulduğumuz teknik ve klinik başarı oranı literatürdeki çalışmalarla uyumludur. Kolonik metal stentlerin yüksek teknik ve klinik başarıları ve düşük komplikasyon oranları, bu stentleri uygun endikasyonu olan hastalarda önemli bir tedavi modalitesi haline getirmiştir. Bu nedenle sadece kolorektal kanserde değil, anastomoz darlıklarında ve kaçaklarında da kolonik metal stentlerin önemli bir tedavi metodu olduğu akılda tutulmalıdır.

\section{KAYNAKLAR}

1. Kochar R, Shah N. Enteral stents: from esophagus to colon. Gastrointest Endosc 2013;78:913-8.

2. ASGE Technology Committee, Varadarajulu S, Banerjee S, Barth B, et al. Enteral stents. Gastrointest Endosc 2011;74:455-64.

3. Srinivasan N, Kozarek RA. Stents for colonic strictures: Materials, designs, and more. Tech Gastrointest Endosc 2014;16:100-7.

4. vanHooft JE, vanHalsema EE, Vanbiervliet G, et al; European Society of Gastrointestinal Endoscopy (ESGE). Self-expandable metal stents for obstructing colonic and extracolonic cancer: European Society of Gastrointestinal Endoscopy (ESGE) Clinical Guideline. Gastrointest Endosc 2014;80:747-61

5. Endoscopy and Cancer Committee of the French Society of Digestive Endoscopy (SFED) and the French Federation of Digestive Oncology (FFCD). Manfredi S, Sabbagh C, Vanbiervliet G, et al. Place of colorectal stents in therapeutic management of malignant large bowel obstructions. Endoscopy 2014;46:546-52

6. Amrani L, Ménard C, Berdah S, et al. From iatrogenic digestive perforation to complete anastomotic disunion: endoscopic stenting as a new concept of "stent-guided regeneration and re-epithelialization". Gastrointest Endosc 2009;69:1282-7.

7. DiMaio CJ, Dorfman MP, Gardner GJ, et al. Covered esophageal self expandable metal stents in the nonoperative management of postoperative colorectal anastomotic leaks. Gastrointest Endosc 2012;76:431-5.

8. Cooper CJ, Morales A, Othman MO. Outcomes of the use of fully covered esophageal self-expandable stent in the management of colorectal anastomotic strictures and leaks. Diagn Ther Endosc 2014;2014:187541.

9. Lamazza A, Sterpetti AV, De Cesare A, et al. Endoscopic placement of self-expanding stents in patients with symptomatic anastomotic leakage after colorectal resection for cancer: long-term results. Endoscopy 2015;47:270-2

10. Loras Alastruey C, Andújar Murcia X, Esteve Comas M. The role of stents in the treatment of Crohn's disease strictures. Endosc Int Open 2016;4:E301-8.

11. Currie A, Christmas C, Aldean H, et al. Systematic review of self-expanding stents in the management of benign colorectal obstruction. Colorectal Dis 2014;16:239-45.

12. Caruso A, Conigliaro R, Manta R, et al. Fully covered self-expanding metal stents for refractory anastomotic colorectal strictures. Surg Endosc 2015;29:1175-8.

13. Cereatti F, Fiocca F, Dumont JL, et al. Fully covered self-expandable metal stent in the treatment of postsurgical colorectal diseases: outcome in 29 patients. Therap Adv Gastroenterol 2016;9:180-8.

14. Vanbiervliet G, Bichard P, Demarquay JF, et al; Research Committee of the French Society of Digestive Endoscopy (SFED). Fully covered self-expanding metal stents for benign colonic strictures. Endoscopy 2013;45:35-41. 